\title{
Fabrication of Smart Shape Memory Fluorosilicon Thermoplastic Vulcanizates: The Effect of Interfacial Compatibility and Tiny Crystals
}

\author{
Jianfeng Fan, Mengwen Yan, Jiarong Huang, Liming Cao, Yukun Chen*
}

Lab of Advanced Elastomer, School of Mechanical and Automotive Engineering, South China University of Technology, 381 Wushan Road, Tianhe District, Guangzhou 510640, China

The supporting information included 4 pages, 4 figures and 1 table

SI.1 The surface tension of the main experimental raw materials

SI.2 Mechanical properties of PVDF/FKM/SR blends

SI.3 DSC characterization

SI.4 SEM images of the cryogenically fractured surface

SI.5 The number-average particle size and their corresponding size distributions 


\section{SI.1 The surface tension of the main experimental raw materials}

Table S1. The surface tension of the main experimental raw materials

\begin{tabular}{cccc}
\hline & $\gamma^{\mathrm{d}}$ & $\gamma^{\mathrm{p}}$ & $\gamma$ \\
\hline SR & 3.5 & 20.5 & 24 \\
FKM & 1.4 & 29.1 & 30.5 \\
PVDF & 3.09 & 44.32 & 47.41
\end{tabular}

in which $\mathrm{A}$ and $\mathrm{B}$ are two components, $\gamma$ represents their surface tension, and $\gamma=$ $\gamma^{\mathrm{d}}$ (dispersive) $+\gamma^{\mathrm{p}}$ (polar). Further, the harmonic-mean equation ${ }^{1}$ (equation $\mathrm{S} 1$ ) is used to calculating the interfacial tension $\left(\gamma_{\mathrm{AB}}\right)$ between the component $\mathrm{A}$ and $\mathrm{B}$ of a blend.

$$
\gamma_{\mathrm{AB}}=\gamma_{\mathrm{A}}+\gamma_{\mathrm{B}}-2\left(\sqrt{\gamma_{\mathrm{A}}^{\mathrm{d}} \times \gamma_{\mathrm{B}}^{\mathrm{d}}}+\sqrt{\gamma_{\mathrm{A}}^{\mathrm{p}} \times \gamma_{\mathrm{B}}^{\mathrm{p}}}\right)
$$

\section{SI.2 Mechanical properties of PVDF/FKM/SR blends}
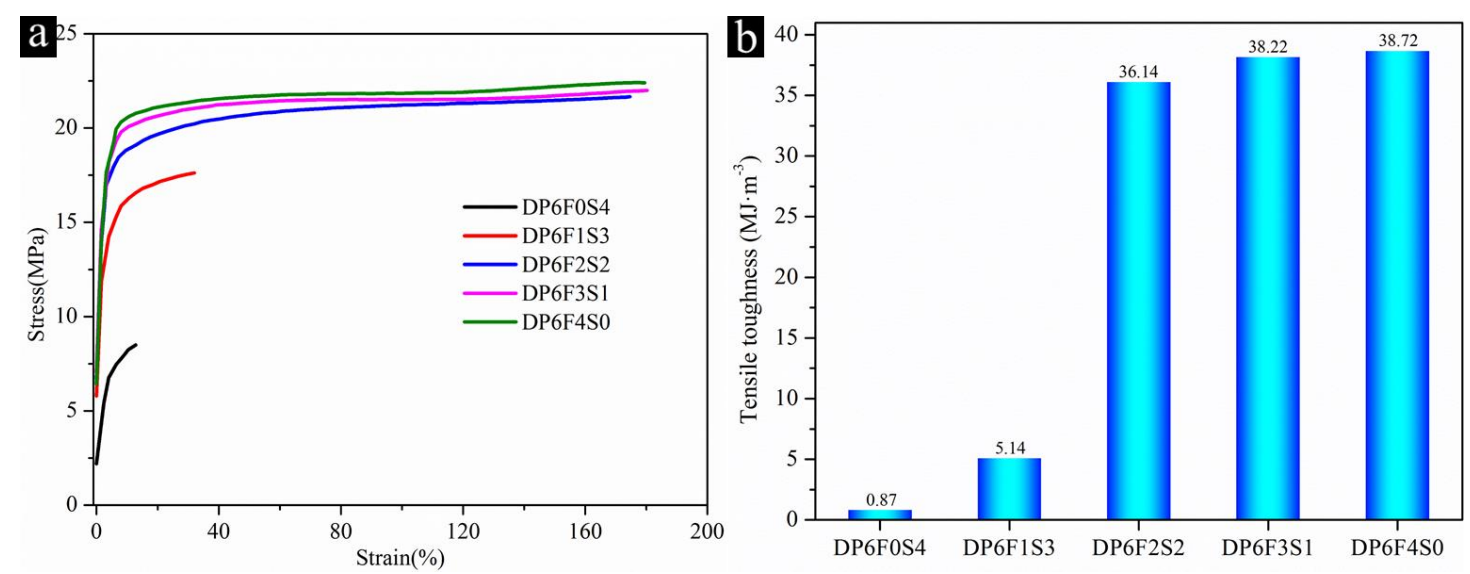

Figure S1. Typical stress-strain curves (a) and tensile toughness (b) of PVDF/FKM/SR blends with different shell-core ratio. 
SI.3 DSC characterization
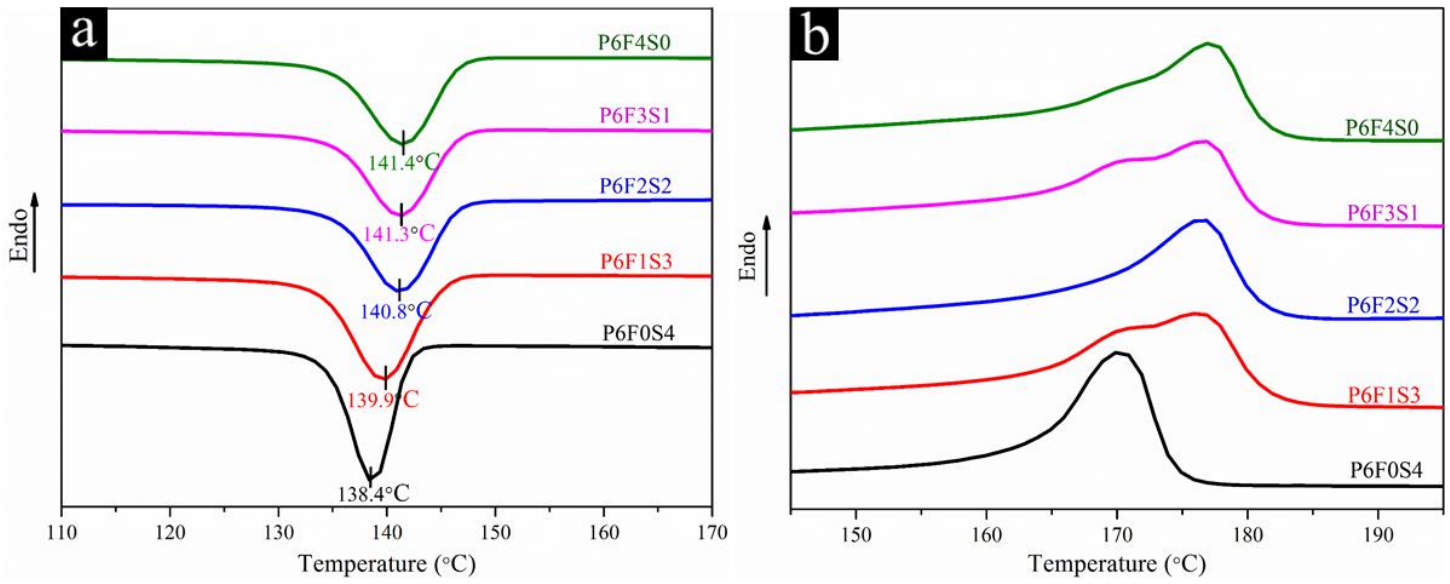

Figure S2. The nonisothermal crystallization curves (a) and nonisothermal heating curves (b) of the PVDF/FKM/SR blends with heating/cooling rate of $10^{\circ} \mathrm{C} / \mathrm{min}$.

\section{SI.4 SEM images of the cryogenically fractured surface}
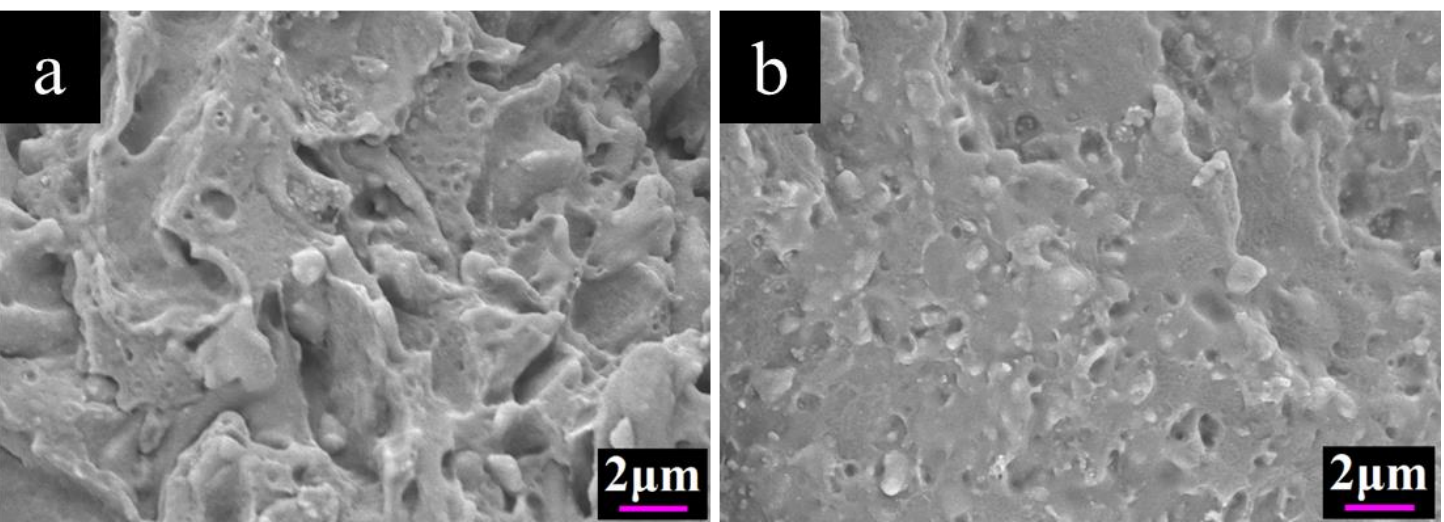

Figure S3. SEM images of the cryogenically fractured surface of the P6F1S3 (a) and P6F3S1 (b). 
SI.5 The number-average particle size and their corresponding size distributions

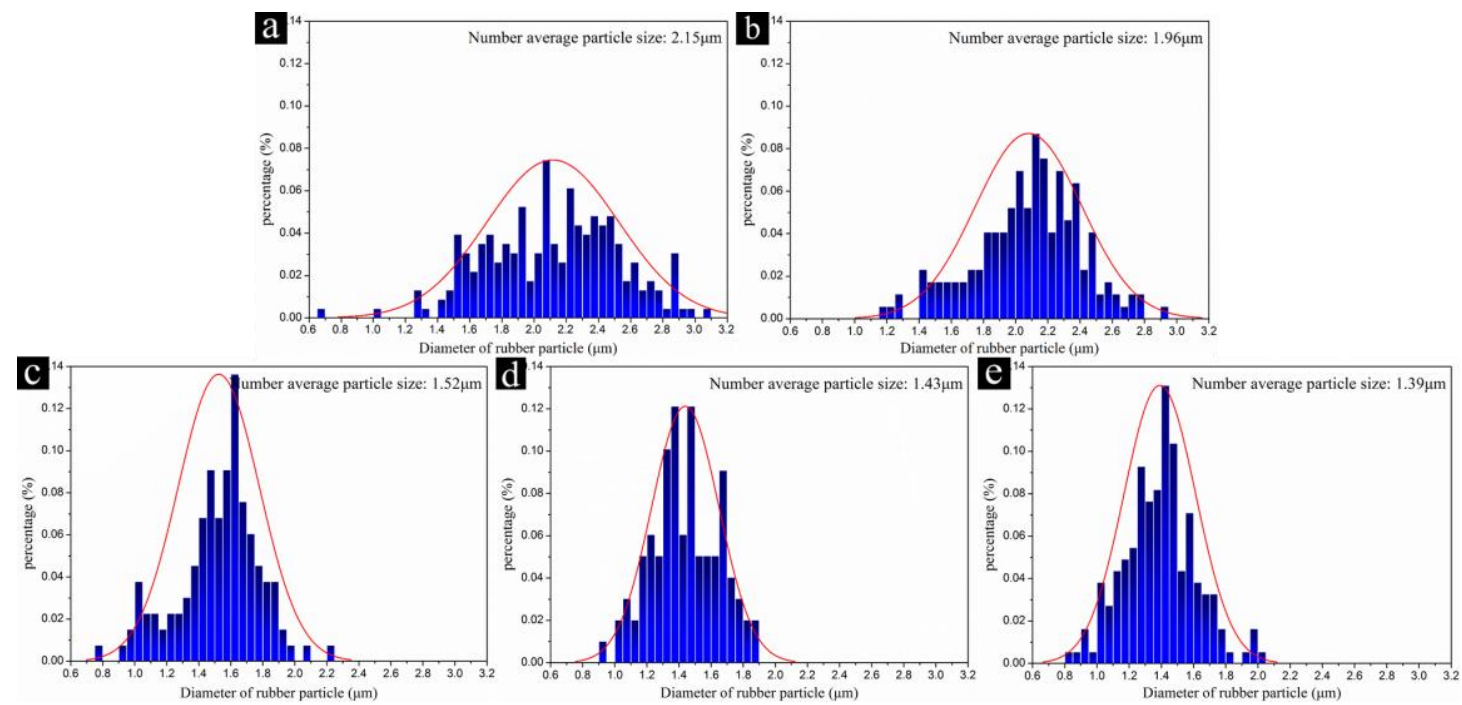

Figure S4. The number-average particle size and their corresponding size distributions based on SEM images of the etched surface by DMF: (a) P6F0S4; (b) P6F1S3; (c) P6F2S2; (d) P6F3S1; (e) P6F4S0.

At least 300 particles from five independent SEM images were counted by the Image-Pro Plus 4.5 software, charting their corresponding size distributions as shown in Figure $\mathrm{S} 4$, and calculating $D_{n}$ using the equation (1).

\section{Reference}

[1] Liu, Y. T.; Cao, L. M.; Yuan, D. S.; Chen, Y. K. Design of super-tough co-continuous $\mathrm{PLA} / \mathrm{NR} / \mathrm{SiO}_{2}$ TPVs with balanced stiffness-toughness based on reinforced rubber and interfacial compatibilization. Compos. Sci. Technol. 2018, 165, 231-239. 